\title{
Hematologia de tilápia-do-nilo alimentada com suplemento à base de algas frente a desafios de estresse agudo e crônico
}

\author{
[Hematology of Nile tilapia fed supplementation with algae challenged by acute and chronic stress] \\ F. Garcia ${ }^{1}$, S.H.C. Schalch ${ }^{1}$, E.M. Onaka ${ }^{2}$, F.S. Fonseca ${ }^{2}$, M.P. Batista ${ }^{3}$
}

\begin{abstract}
${ }^{1}$ Pólo Regional Noroeste Paulista - Agência Paulista de Tecnologia dos Agronegócios, APTA - Secretaria de Agricultura e Abastecimento do Estado de São Paulo - SAA - Votuporanga, SP

${ }^{2}$ Centro do Pescado Continental - Agência Paulista de Tecnologia dos Agronegócios - Instituto de Pesca - APTA Secretaria de Agricultura e Abastecimento do Estado de São Paulo - SAA - Votuporanga, SP

${ }^{3}$ UNIFEV - Centro Universitário de Votuporanga, no curso de Ciência Biológicas - Votuporanga, SP
\end{abstract}

\begin{abstract}
RESUMO
Avaliou-se o efeito da suplementação com produto à base de algas, ergosan, em dietas para tilápias Oreochromis niloticus, submetidas ao desafio de estresse agudo e crônico sobre as características hematológicas. O ensaio foi realizado com tilápias jovens $(15 \mathrm{~g})$ em caixas de $500 \mathrm{~L}$ de capacidade de estocagem, em sistema com recirculação da água, com fluxo constante, na densidade de 35 peixes por caixa. Os peixes foram alimentados com as dietas-teste durante 10 dias e, ao final deste período, voltaram a receber dieta isenta de ergosan, quando foram submetidos aos desafios de estresses agudo e crônico. Utilizou-se o delineamento inteiramente ao acaso (DIC), em esquema fatorial 4x2, sendo testados: quatro porcentagens do suplemento $0 ; 0,25 ; 0,5$ e $1 \%$ de ergosan na ração em dois tipos de estresse, agudo e crônico. Os resultados permitem concluir que os estímulos de estresse aplicados foram capazes de provocar alterações fisiológicas nos peixes, incluindo redução no número de eritrócitos, eritroblastos e leucócitos e aumento do volume corpuscular médio dos eritrócitos. A administração oral de $1 \%$ de suplemento durante 10 dias para juvenis de tilápia-do-nilo, em condição de estresse agudo, induz o incremento de $69 \%$ no número de trombócitos circulantes.
\end{abstract}

Palavras-chave: ergosan, Oreochromis niloticus, peixe, temperatura

\begin{abstract}
The aim of this trial was to evaluate hematological parameters of tilapia Oreochromis niloticus fed diet supplemented with ergosan, an algae product, in a chronic and acute stress challenge. The experiment was carried out with juvenile tilapias Oreochromis niloticus (15g), in 500 L-tanks linked at a water recirculation system, with constant water flow and controlled temperature, stoking 35 fish/tank. Fish were fed test-diets during 10 days and, at the end of this period, received a diet without supplementation, when they were submitted to chronic and acute stress challenge. Fish were distributed in a completely randomized design, in a factorial scheme 4x2: four supplement levels $(0 ; 0.25 ; 0.5$ and 1\% supplement in the diet) with two stresses (chronic and acute). Results showed the stress stimulus applied were able to induce physiologic changes on fish, including reduction of erythrocytes, erythroblasts and leucocytes number and increasing medium corpuscular volume. Oral administration of 1\% supplement during 10 days for Nile tilapia juveniles under acute stress induces a higher thrombocyte number (69\%) in the blood.
\end{abstract}

Keywords: ergosan, fish, Oreochromis niloticus, temperature

Recebido em 11 de março de 2011

Aceito em 13 de setembro de 2011

E-mail: fgarcia@apta.sp.gov.br 


\section{INTRODUÇÃO}

A tilápia tem se apresentado como uma espécie de grande interesse no mercado devido à sua carne de excelente sabor e ausência de espinhas no filé (Silva, 1999), por ser uma espécie cosmopolita de prolificidade elevada, hábito alimentar onívoro, rápido crescimento e rusticidade.

No Brasil, um dos principais gargalos na produção de tilápias em tanques-rede é o controle sanitário, que tem como objetivo reduzir as elevadas taxas de mortalidade (Anuário..., 2001). Neste contexto, os imunomoduladores e imunoestimulantes podem representar grandes avanços na aquicultura, pois sua utilização melhora as respostas imunes não específicas a uma ampla variedade de patógenos e aumenta a resposta imune em animais debilitados ou com o sistema imune em desenvolvimento (larvas e alevinos) (Bricknell e Dalmo, 2005).

O ergosan é um complemento alimentar composto por algas marinhas secas e extratos vegetais de Laminaria digitata e Ascophyllum nodosum. Os ingredientes ativos, incluindo alginas e polissacarídeos, são conhecidos por melhorar os sistemas de defesa natural do peixe. É um produto natural e aceito como um ingrediente alimentar. Como a maioria dos alginatos, o ergosan contém ácidos poliurônicos, que atuam como catalisadores da absorção de oxigênio pelas células, melhorando sua vitalidade (Miles et al., 2001). Este produto apresenta um amplo e conhecido efeito imunoestimulador em salmão-do-atlântico Salmo salar (Hall, 1998).

Bagni et al. (2005) verificaram que a suplementação com $0,5 \%$ deste suplemento na ração de sea bass Dicentrarchus Labrax por 15 dias elevou a atividade do sistema complemento durante o período em que os peixes estavam recebendo a suplementação e que a produção de lisozimas foi melhorada 30 dias após a suplementação. Quanto aos aspectos de produção, para juvenis de tilápia-do-nilo Oreochromis niloticus, a suplementação alimentar com ergosan melhorou o consumo de ração e o ganho de peso em uma relação doseresposta linear (Garcia et al., 2009). Jalali et al. (2009) observaram melhora nos parâmetros de desempenho e hematologia em esturjão Huso huso alimentado com ergosan.

Em estudo anterior, Marino (2003) relatou o efeito imunomodulador dos ácidos algínicos, fornecidos na dieta, na imunidade não específica de sea bass sob condição estressante. A rota de administração do produto mais eficiente parece ser a oral, pois Peddie et al. (2002) descreveram a ineficiência imunomoduladora do produto quando administrado intraperitonealmente em truta arco-íris Oncorhynchus mykiss.

Sendo assim, o objetivo deste ensaio foi avaliar o efeito da suplementação com ergosan em dietas para tilápias Oreochromis niloticus, submetidas a desafio de estresses agudo e crônico, nos parâmetros hematológicos.

\section{MATERIAL E MÉTODOS}

Foram utilizadas tilápias $O$. niloticus jovens, com peso inicial de aproximadamente $15 \mathrm{~g}$. O ensaio foi conduzido no setor de piscicultura da APTA, em Votuporanga, SP, em caixas de 500L de capacidade de estocagem, em sistema com recirculação da água, com fluxo constante, na densidade de 35 peixes por caixa. Os peixes foram aclimatados por um período inicial de sete dias. Após a aclimatação, foram alimentados com as dietas-teste durante 10 dias e, ao final deste período, voltaram a receber ração isenta do produto e foram submetidos aos desafios de estresses agudo e crônico.

Utilizou-se o delineamento inteiramente ao acaso, em esquema fatorial $4 \times 2$, sendo testados: quatro porcentagens de suplemento à base de algas (Aquavac Ergosan ${ }^{\circledR}$ - Intervet Schering Plough Animal Health) 0; 0,25; 0,5 e 1\% do produto na ração, em dois tipos de estresse, agudo e crônico, com três repetições.

A dieta-base utilizada continha $32,5 \%$ de proteína, e a composição está apresentada na Tab. 1. O suplemento foi incorporado aos ingredientes moídos e depois a mistura foi peletizada a frio.

O estresse constou de aumento de temperatura da água de $26^{\circ} \mathrm{C}$ para $33^{\circ} \mathrm{C}$, sendo que os peixes foram mantidos à temperatura de $33^{\circ} \mathrm{C}$ durante cinco dias e, no quinto dia, aplicou-se o manejo de captura: todos os peixes de cada caixa foram capturados com rede e mantidos fora da água por 
dois minutos. Este método simulou uma forma de estresse comum na piscicultura e já foi realizado por Davis e Schreck (1997) e Martins et al. (2004). Consideraram-se estresse agudo as primeiras 24 horas em que os peixes foram mantidos à temperatura elevada, e estresse crônico o quinto dia à temperatura elevada, após o manejo de captura.

Tabela 1. Composição e análise bromatológica da dieta-base utilizada no ensaio com tilápia-do-nilo

\begin{tabular}{lc}
\hline \multicolumn{1}{c}{ Ingredientes } & $\%$ \\
\hline Farinha de peixe (50\%PB) & 14,41 \\
Levedura (cana-de-açúcar) & 4,00 \\
Farelo de soja & 20,12 \\
Glúten de milho (60\%PB) & 9,52 \\
Milho & 22,30 \\
Farelo de trigo & 22,30 \\
Óleo de peixe & 1,70 \\
Óleo de soja & 2,40 \\
Suplemento min. e vit.* & 1,00 \\
Sal (NaCl) & 0,15 \\
BHT & 0,15 \\
Calcário & 0,63 \\
Fosfato bicálcico & 1,98 \\
L-lisina 78 & 1,09 \\
DL-metionina 98 & 0,14 \\
Caulin & 0,15
\end{tabular}

\begin{tabular}{lc} 
Composição & \\
Matéria seca & 92,95 \\
Proteína bruta & 32,50 \\
Proteína digestível & 29,20 \\
Extrato etéreo & 7,050 \\
Matéria mineral & 8,90 \\
Energia digestível & 3,00 \\
\hline *Premix: Vitaminas: A=2200,00UI, D3=1600,00UI
\end{tabular}

*Premix: Vitaminas: $A=2200,00 U I, D 3=1600,00 U I$, $\mathrm{E}=17,00 \mathrm{UI}, \quad \mathrm{K}=2,50 \mathrm{mg}, \quad \mathrm{B} 1=2,50 \mathrm{mg}, \mathrm{B} 2=4,00 \mathrm{mg}$, B6 $=2,00 \mathrm{mg}, \quad B 12=30,00 \mathrm{mcg}, \quad \mathrm{C}=100,00 \mathrm{mg}$, Ácido fólico $=1,00 \mathrm{mg}$, ácido pantoténico $=15,00 \mathrm{mg}$, biotina $=0,10 \mathrm{mg}, \quad$ niacina $=50,00 \mathrm{mg}, \quad$ colina $=4,50 \mathrm{~g}$, cobalto $=0,03 \mathrm{mg}, \quad$ cobre $=7,50 \mathrm{mg}$, ferro $=50,00 \mathrm{mg}$, iodo $=2,00 \mathrm{mg}$, manganês $=50,00 \mathrm{mg}$, selênio $=0,07 \mathrm{mg}$, zinco $=80,00 \mathrm{mg}$, antioxidante $=125,00 \mathrm{~g}$

Para a análise hematológica, alíquotas de sangue foram colhidas por punção do vaso caudal de dois exemplares por caixa, a cada coleta, utilizando-se seringas contendo EDTA (10\%). As variáveis hematológicas foram analisadas com 24h à temperatura da água elevada (estresse agudo) e cinco dias depois, logo após o manejo de captura (estresse crônico), sendo as seguintes: glicemia, hematócrito, contagem de eritrócitos, volume corpuscular médio (VCM), contagem total de leucócitos, eritroblastos e leucócitos. As taxas de mortalidade e morbidade, observações de ocorrência e caracterização dos sinais clínicos foram realizadas diariamente durante $o$ experimento e até uma semana após o desafio.

Os dados foram submetidos à análise de variância a 5\% de probabilidade. Quando os resultados foram significativos, foi aplicado o teste de Tukey para comparação de médias.

\section{RESULTADOS E DISCUSSÃO}

A taxa de mortalidade durante o experimento foi menor que $5 \%$, e não houve diferença estatística entre os tratamentos $(\mathrm{P}>0,05)$. Na Tab. 2, estão apresentados os resultados da estatística obtida da análise de variância das características hematológicas de tilápias-do-nilo alimentadas com suplemento à base de algas e submetidas aos estresses crônico e agudo.

Ao final dos cinco dias, sob condição estressante (estresse crônico), em que os peixes foram mantidos em temperatura acima da recomendada para a espécie, houve redução $(\mathrm{P}<0,05)$ no número de eritrócitos, eritroblastos e leucócitos, enquanto o volume corpuscular médio dos eritrócitos aumentou $(\mathrm{P}<0,05)$ (Fig. 1). Pelas alterações hematológicas ocorridas no estresse, verifica-se que o desafio foi suficiente para provocar respostas fisiológicas no peixe.

$\mathrm{O}$ aumento da temperatura da água reduziu o oxigênio dissolvido à concentração próxima a $2 \mathrm{mg} / \mathrm{L}$, considerada inferior à recomendada para o cultivo de peixes (Sipaúba-Tavares, 1994). De acordo com Baldisserotto (2002), a baixa disponibilidade de oxigênio da água é um dos fatores ambientais mais estressantes para os peixes e requer ajustes adaptativos em todos os níveis de organização biológica. Val et al. (2000) verificaram que Lipossarcus pardalis em hipóxia ambiental apresenta elevação no VCM, sugerindo aumento na capacidade de carreamento de oxigênio. Há evidências de que a temperatura ativa a eritropoiese e a liberação de eritrócitos jovens dos compartimentos de estocagem (Houston e Murad, 1995) na tentativa de aumentar a demanda de oxigênio para os tecidos (Lecklin e Nikinmaa, 1998). Entretanto, após o estresse crônico, o peixe, provavelmente, perde a capacidade de manter a eritropoise, e a eficiência no carreamento do oxigênio passa a ser alcançada pelo aumento no volume dos eritrócitos (Fig. 1). 
Tabela 2. Estatística obtida na análise de variância e resultados de hematologia (média e desvio-padrão) de Oreochromis niloticus alimentadas com dietas contendo 0;0,25;0,5 e 1\% de suplemento na ração frente aos estresses agudo e crônico

\begin{tabular}{|c|c|c|c|c|}
\hline & \multicolumn{2}{|c|}{ F suplemento } & F estresse & $\begin{array}{c}\text { F suplemento } \mathrm{x} \\
\text { estresse }\end{array}$ \\
\hline $\mathrm{Ht}(\%)$ & \multicolumn{2}{|l|}{$1,32^{\mathrm{NS}}$} & $0,045^{\mathrm{NS}}$ & $1,59^{\mathrm{NS}}$ \\
\hline Eritrócito $\left(\times 10^{6} / \mathrm{mm}^{3}\right)$ & \multicolumn{2}{|l|}{$1,78^{\mathrm{NS}}$} & $25,16 * *$ & $1,49^{\mathrm{NS}}$ \\
\hline Eritroblasto $\left(\times 10^{5} / \mathrm{mm}^{3}\right)$ & \multicolumn{2}{|l|}{$0,74^{\mathrm{NS}}$} & $48,57 * *$ & $0,71^{\mathrm{NS}}$ \\
\hline Glicemia (g/dL) & \multicolumn{2}{|l|}{$0,99^{\mathrm{NS}}$} & $2,90^{\mathrm{NS}}$ & $0,35^{\mathrm{NS}}$ \\
\hline VCM (fL) & \multicolumn{2}{|l|}{$0,26^{\mathrm{NS}}$} & $16,67 * *$ & $0,76^{\mathrm{NS}}$ \\
\hline Leucócito $\left(\times 10^{4} / \mathrm{mm}^{3}\right)$ & \multicolumn{2}{|l|}{$2,06^{\mathrm{NS}}$} & $4,30 *$ & $0,16^{\mathrm{NS}}$ \\
\hline \multirow[t]{3}{*}{ Trombócito $\left(\times 10^{3} / \mathrm{mm}^{3}\right)$} & \multicolumn{2}{|l|}{$5,21 * *$} & $0,80^{\mathrm{NS}}$ & $6,44 * *$ \\
\hline & \multicolumn{4}{|c|}{ Ergosan } \\
\hline & 0 & 0,25 & 0,5 & 1,0 \\
\hline $\mathrm{Ht}(\%)$ & $36,50 \pm 7,79$ & $38,92 \pm 5,04$ & $36,38 \pm 7,25$ & $37,88 \pm 7,98$ \\
\hline Eritrócito $\left(\times 10^{6} / \mathrm{mm}^{3}\right)$ & $1,03 \pm 0,34$ & $1,16 \pm 0,41$ & $1,03 \pm 0,32$ & $1,21 \pm 0,30$ \\
\hline Eritroblasto $\left(\times 10^{5} / \mathrm{mm}^{3}\right)$ & $0,52 \pm 0,34$ & $0,85 \pm 1,13$ & $0,62 \pm 0,46$ & $0,69 \pm 0,42$ \\
\hline Glicemia (g/dL) & $25,27 \pm 9,76$ & $29,60 \pm 10,94$ & $20,42 \pm 5,81$ & $28,08 \pm 16,70$ \\
\hline VCM (fL) & $3,87 \pm 1,55$ & $3,93 \pm 2,07$ & $3,93 \pm 1,70$ & $3,26 \pm 0,77$ \\
\hline Leucócito $\left(\times 10^{4} / \mathrm{mm}^{3}\right)$ & $2,32 \pm 0,99$ & $3,37 \pm 0,93$ & $2,67 \pm 1,13$ & $2,63 \pm 0,76$ \\
\hline Trombócito $\left(\times 10^{3} / \mathrm{mm}^{3}\right)$ & $5,25 \pm 2,70 b$ & $6,80 \pm 3,6 b$ & $8,24 \pm 3,5 \mathrm{ab}$ & $8,89 \pm 5,5 \mathrm{a}$ \\
\hline
\end{tabular}

Ht - hematócrito, VCM - volume corpuscular médio.

$* \mathrm{P}<0,05 ; * * \mathrm{P}<0,01 ; \mathrm{NS}$ - não significativo.

Letras diferentes na linha indicam diferença estatística entre os valores.

Todas as variáveis apresentam F Levene para homogeneidade de variância $>0,05$ e $\mathrm{X}^{2}$ para normalidade (Shapiro Wilks) $>0,05$.
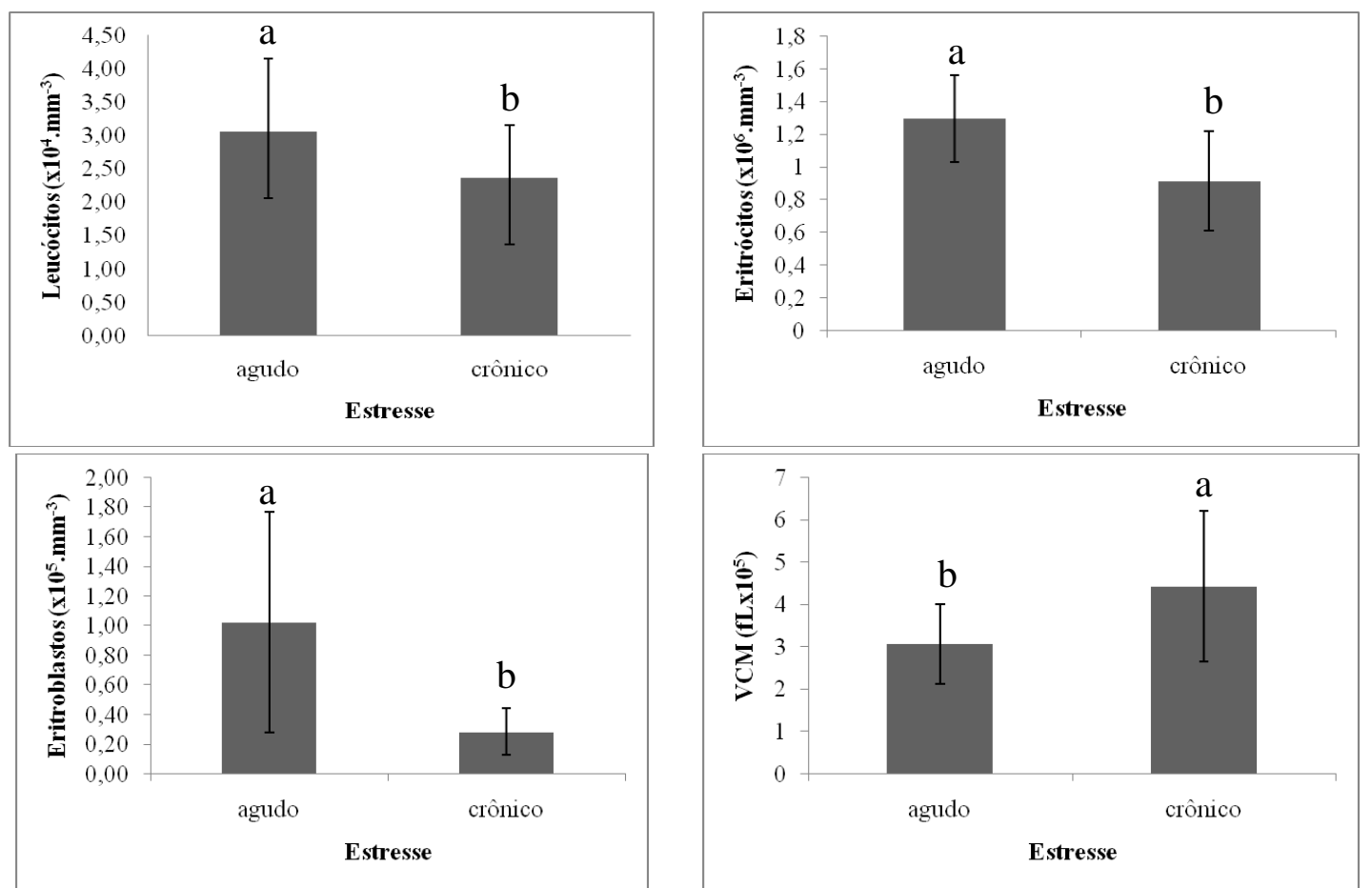

Figura 1. Médias da contagem de leucócitos, eritrócitos e eritroblastos e do volume corpuscular médio (VCM) de tilápias-do-nilo Oreochromis niloticus, submetidas a dois tipos de estresse - agudo e crônico. 
No presente estudo, verificou-se a liberação de eritroblastos e leucócitos nas primeiras 24 horas de estresse e, na segunda análise hematológica, as reduções no número destas células levam à hipótese de exaustão fisiológica do peixe, descrita por Selye (1950): as alterações morfológicas, bioquímicas e fisiológicas resultantes do estresse constituem a síndrome geral de adaptação (SGA), composta pela fase de alarme, quando o organismo sente o estímulo estressante; fase de resistência, na qual o organismo sofre modificações adaptativas, atingindo novo patamar de equilíbrio; e fase de exaustão, em que o organismo perde a capacidade de adaptação com quebra da homeostase orgânica. A partir desse momento, os animais sofrem profundas alterações fisiológicas e bioquímicas, sujeitos frequentemente às enfermidades parasitárias e infecciosas. Em estudo com sea bass, Bagni et al. (2005) descreveram uma drástica supressão tanto nas características imunes inatas quanto na resposta adquirida durante o período de inverno, quando a temperatura da água foi reduzida a índices abaixo do recomendado para a espécie.

Houve interação de adição do suplemento na dieta versus tipo de estresse aplicado (agudo e crônico) na contagem de trombócitos da tilápiado-nilo $(\mathrm{P}<0,01) \quad$ (Tab. 2), sendo que a suplementação com $1 \%$ de suplemento interferiu na resposta ao estresse agudo, provocando aumento de $69 \%$ no número de trombócitos circulantes (Fig. 2). Os resultados de Bagni et al. (2005) revelaram o potencial do ácido algínico em ativar as respostas imunes inatas de sea bass e, particularmente sob condições de imunossupressão relacionada a estresse ambiental.

Esta resposta é interessante, pois, além da conhecida função de coagulação do sangue, estudo recente da ultraestrutura de trombócitos de Colossoma macropomum revelou que estas células apresentam modificações morfológicas, como elevado número de aglomerados de glicogênio, numerosas projeções citoplasmáticas da superfície celular, além de vesículas formadas no citoplasma das células, o que sugere a função fagocítica da célula. Desta forma, os autores afirmaram que os trombócitos de peixes são células que formam uma das barreiras protetoras e podem ser consideradas verdadeiras células digestoras em peixes, pois removem destroços celulares da circulação por fagocitose (TavaresDias et al., 2007).

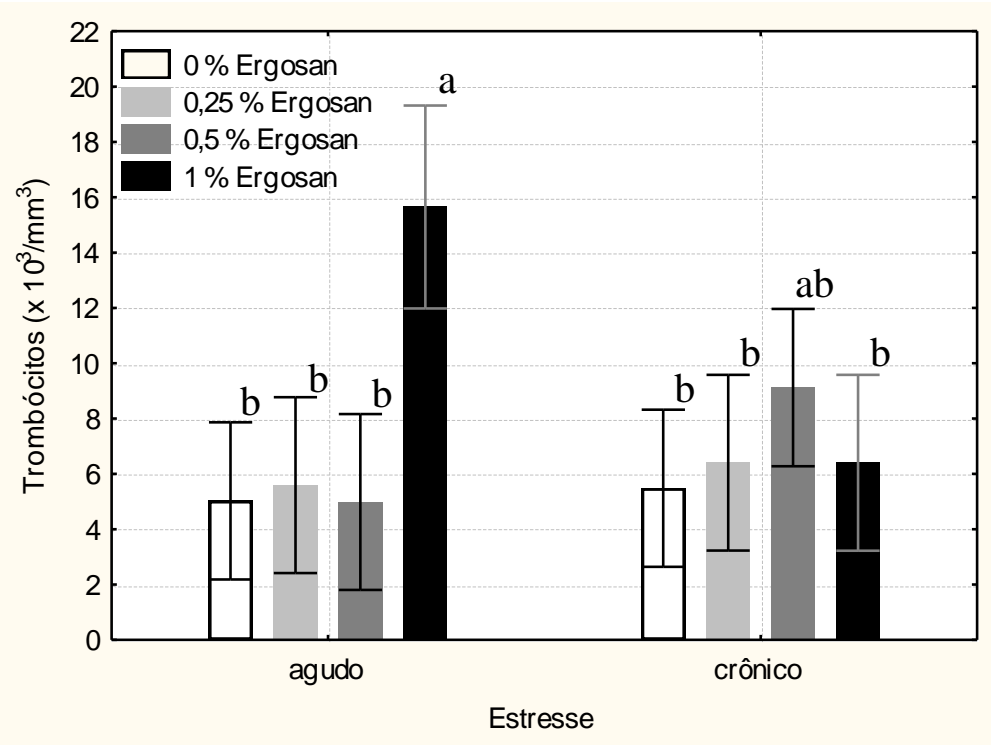

Figura 2. Médias da interação do nível de suplemento oferecido às tilápias-do-nilo Oreochromis niloticus versus tipo de estresse - agudo e crônico - na contagem de trombócitos circulantes. 
Corroborando com esta afirmação, Bozzo et al. (2007) demonstram que as células predominantes no exudato inflamatório de Piaractus mesopotamicus induzido por lamínula com tioglicolato, Aeromonas hydrophila e LPS foram trombócitos, sugerindo que essas células, juntamente com os leucócitos, apresentam funções hemostáticas, agindo também como célula de defesa. Em estudos com essa mesma espécie de peixe, Garcia et al. (2007) relatam que, semelhantemente à resposta encontrada com a suplementação testada, a vitamina $\mathrm{C}$ induziu a um aumento de trombócitos circulantes, em uma relação dose-resposta.

\section{CONCLUSÕES}

A administração oral de $1 \%$ do suplemento durante 10 dias para juvenis de tilápia-do-nilo, em condição de estresse agudo, induz a um maior número de trombócitos circulantes.

\section{REFERÊNCIAS}

ANUÁRIO DA PECUÁRIA BRASILEIRA. São Paulo: FNP Consultoria \& Comércio, 2001.

BAGNI, M.; ROMANO, N.; FINOI, M.G. et al. Short- and long-term effects of a dietary yeast bglucan (Macrogard) and alginic acid (Ergosan) preparation on immune response in sea bass (Dicentrarchus labrax). Fish Shellfish Immunol., v.18, p.311-325, 2005.

BALDISSEROTTO, B. Fisiologia de peixes aplicada à piscicultura. Santa Maria: UFSM, 2002. 212p.

BOZZO, F.R.; MORAES, J.R.E.; MORAES, F.R. et al. Kinetics of cellular component in inflammatory response induced by different stimuli in the swim bladder of pacu, Piaractus mesopotamicus Holmberg 1887 (Characidae). J. World Aquac. Soc., v.38, p.302-308, 2007.

BRICKNELL, I.; DALMO, R.A. The use of immunostimulants in fish larval aquaculture. Fish Shellfish Immunol., v.19, p.457-472, 2005.

DAVIS, E.L.; SCHRECK, C.B. The energetic response to handling stress in juvenile coho salmon. Transactions Am. Fish. Soc., v.126, p.248- 258, 1997.
GARCIA, F.; ABIMORAD, E.G.; SCHALCH, S.H.C. et al. Desempenho produtivo de tilápiasdo-nilo alimentadas com suplemento alimentar à base de algas. Bioikos, v.23, p.83-89, 2009.

GARCIA, F.; PILARSKI, F.; ONAKA, E.M. et al. Hematology of Piaractus mesopotamicus fed diets supplemented with vitamins $\mathrm{C}$ and $\mathrm{E}$, challenged by Aeromonas hydrophila. Aquaculture, v.271, p.39-46, 2007.

HALL, T.E. In vitro and in vivo studies into the influences of an algal extract on non-specific immune parameters in the Atlantic salmon, Salmo salar. 1998 Thesis (Master of Sciences) University of Plymouth, UK.

HOUSTON, A.H.; MURAD, A. Erythrodynamics in fish - recovery of the goldfish Carassius auratus from acute anemia. Can. J. Zool., v.73, p.411418, 1995.

JALALI, M.A.; AHMADIFAR, E.; SUDAGAR, M. et al. Growth efficiency, body composition, survival and haematological changes in great sturgeon (Huso huso Linnaeus, 1758) juveniles fed diets supplemented with different levels of Ergosan. Aquac. Res., v.40, p.804-809, 2009.

LECKLIN, T.; NIKINMAA, M. Erythropoiesis in artic charr is not stimulated by anemia. J. Fish Biol., v.53, p.1169-1177, 1998.

MARINO, G. Measurement of chemical, clinical and non-specific immune parameters for evaluation of welfare in sea bass Dicentrarchus labrax. Rome, Italy: Ministry of Agriculture, 2003. (Report of the project 5C/68).

MARTINS, M.L.; PILARSKI, F.; ONAKA, E.M. et al. Hematologia e resposta inflamatória aguda em Oreochromis niloticus (Osteichthyes: Cichlidae) submetida aos estímulos único e consecutivo de estresse de captura. Bol. Inst. Pesca, v.30, p.71-80, 2004.

MILES, D.J.C.; POLCHANA, J.; LILLEY, J.H. et al. Immunostimulation of striped snakehead Channa striata against epizootic ulcerative syndrome. Aquaculture, v.195, p.1-15, 2001.

PEDDIE, S.; ZOU, J.; SECOMBES, C.J. Immunostimulation in the rainbow trout (Oncorhynchus mykiss) following intraperitoneal administration of Ergosan. Vet. Immunol. Immunophatol., v.86, p.101-113, 2002. 
SELYE, H. Stress and the general adaptation syndrome. Brit. Med. J. v.1, p.1383-1392, 1950.

SILVA, M.A.F. O mercado para produtos industrializados da pesca e aquicultura. In: WORKSHOP INTERNACIONAL DE AQUICULTURA 3. 1999. Anais... São Paulo. São Paulo: Gessulli Eventos, 1999, p.18-20.

SIPAÚBA-TAVARES, L.H. Limnologia aplicada à aquicultura. Jaboticabal: Editora Funep, SP, 1994, 72p. (Boletim Técnico $\mathrm{n}^{\mathrm{o}}$ 1).
TAVARES-DIAS, M.; ONO, E.A.; PILARSKI, F. et al. Can thrombocytes participate in the removal of cellular debris in the blood circulation of teleost fish? A cytochemical study and ultrastructural analysis. J. Appl. Ichthyol., v.23, p.709-712, 2007.

VAL, A.L. Organic phosphates in the red blood cells of fish. Comp. Biochem. Physiol., v.125, p.417-435, 2000. 